Review Article

\title{
Diagnostic Value of CK-18, FGF-21, and Related Biomarker Panel in Nonalcoholic Fatty Liver Disease: A Systematic Review and Meta-Analysis
}

\author{
Lei He, ${ }^{1}$ Linfeng Deng, ${ }^{1}$ Quan Zhang, ${ }^{2}$ Jianli Guo, ${ }^{3}$ \\ Jinan Zhou, ${ }^{1}$ Wenjian Song, ${ }^{4}$ and Fahu Yuan ${ }^{4}$ \\ ${ }^{1}$ Department of Blood Transfusion, Tongji Hospital, Tongji Medical College, Huazhong University of Science and Technology, \\ Wuhan, China \\ ${ }^{2}$ Department of Laboratory Medicine, Hubei Provincial Hospital of Integrated Chinese \& Western Medicine, Wuhan, China \\ ${ }^{3}$ Department of Biochemistry and Molecular Biology, School of Basic Medicine, Tongji Medical College, \\ Huazhong University of Science and Technology, Wuhan, China \\ ${ }^{4}$ School of Medicine, Jianghan University, Wuhan, China
}

Correspondence should be addressed to Fahu Yuan; yuanfh@jhun.edu.cn

Received 19 November 2016; Accepted 19 January 2017; Published 23 February 2017

Academic Editor: Kusum Kharbanda

Copyright (C) 2017 Lei He et al. This is an open access article distributed under the Creative Commons Attribution License, which permits unrestricted use, distribution, and reproduction in any medium, provided the original work is properly cited.

Liver biopsy still remains the gold standard for diagnosing nonalcoholic steatohepatitis (NASH), but with limitations. There is an urgent need to develop noninvasive tests that accurately distinguish NASH from simple steatosis. The purpose of this meta-analysis was to evaluate the diagnostic value of serum biomarkers including cytokeratin 18 (CK-18), fibroblast growth factor 21 (FGF-21), and combined biomarker panel (CBP) in the diagnosis of NAFLD, especially NASH. A total of 25 studies met the inclusion criteria. Pooled sensitivity and specificity values for chosen serum markers for diagnosing NASH are as follows: CK-18 (M30), 0.75 and 0.77; CK-18 (M65), 0.71 and 0.77; FGF-21, 0.62 and 0.78; and CBP, 0.92 and 0.85. CBP demonstrated better accuracy with higher sensitivity and specificity than those tested individually. Furthermore, the AUROC of CBP was 0.94 (95\% CI, 0.92-0.96), compared to CK-18 or FGF-21 assay, which showed the most significant ability to distinguish NASH from simple steatosis. The results suggest that increased circulating CK-18 and FGF-21 are associated with NASH and may be used for initial assessment, but not enough. Importantly, CBP is potentially used as accurate diagnostic tools for NASH. Further prospective designed studies are warranted to confirm our findings.

\section{Introduction}

Nonalcoholic fatty liver disease (NAFLD) has become an important public health problem because of its high prevalence and potential progression to severe liver disease. Steatosis, the hallmark feature of NAFLD, refers to excessive amount of lipid in liver, and the processes are linked to lipid metabolism disorders [1-3]. In some individuals, steatosis progresses to nonalcoholic steatohepatitis (NASH), which encompasses spectrum of hepatic pathological changes including inflammation, apoptosis, and fibrosis. Above all, $\mathrm{NASH}$ is a potentially serious condition, because as many as $20 \%$ patients in whom may progress to cirrhosis or hepatocellular carcinoma (HCC) $[4,5]$.

Importantly, NAFLD/NASH as the hepatic entity of the metabolic syndrome may itself pose a risk factor for HCC, even in the absence of cirrhosis. As observed from Ertle et al. [6], patients with NAFLD/NASH-associated HCC exhibited a higher prevalence of metabolic features; $41.7 \%$ of them had no evidence of cirrhosis. Thus, the possibility of HCC derived from noncirrhotic NASH liver would be an even greater impetus for early detection, early prevention, and early treatment. At present, liver biopsy is the gold standard 
for diagnosing NAFLD. There is no doubt that the biopsy for this disease has several important limitations. Firstly, it is an invasive procedure that requires hospitalization. Furthermore, the procedure may result in major complications like hemorrhage and may not be acceptable by some patients. The relative complexity and high costs of the gold standard approach employed for diagnosing the vast majority of NAFLD have urged the field to search for alternative diagnostic methods.

Due to the important limitations of liver biopsy, lots of studies have tried to identify potential novel biomarkers based on the current knowledge of the pathophysiologic mechanisms involved in the progression of NAFLD. The available biomarkers should be simple, repeatable, inexpensive, and accurate for a particular disease process, specifically for distinguishing NASH from simple steatosis, predicting risk of disease progression [7]. Such a test would not only aid clinicians in the identification of patients with $\mathrm{NASH}$, but also allow for noninvasive frequent monitoring of disease progression and response to therapy. Recently, one important review [8] summarized several novel circulating biomarkers including cytokeratin 18 (CK-18), fibroblast growth factor 21 (FGF-21), and related biomarker panel combining more than two kinds of serum proteins. These biomarkers are released into circulation as a consequence of oxidative stress, hepatocyte apoptosis, or inflammation in response to lipid metabolism disorders because of NAFLD. However, how to select the most accurate biomarker is still controversial. To the best of our knowledge, only a few studies focus on synthetic evaluation and identify the diagnostic value of CK$18[9,10]$. Thus, the accurate information in this regard is desperately required. In this work, we performed a systematic review and meta-analysis aiming to summarize the results of published studies regarding the diagnostic effect of the serum biomarkers in NAFLD/NASH.

\section{Methods}

2.1. Data Sources and Searches. Articles published up to September 2016 were extracted from 4 electronic databases: PubMed, Embase, ISI Web of Science, and the Cochrane Library. Two authors (LH and LFD) independently conducted a medical literature search and screening. The following keywords combined with their corresponding $\mathrm{MeSH}$ terms were used: (NAFLD or NASH) and ("Serum biomarkers" or "Cytokeratin 18" or "Fibroblast growth factor 21" or "Combined biomarker"). Additionally, the references cited in retrieved articles were scrutinized by manual search. The final results were reached after discussion.

2.2. Inclusion Criteria. Inclusion criteria were as follows: (1) studies investigated the diagnostic value of serum biomarkers for NAFLD and contained adequate data to construct a $2 \times$ 2 diagnostic table. (2) In each study, the final diagnosis of NAFLD had to be confirmed by histopathology of liver biopsy as the reference standard. (3) The individual study results at least were presented graphically by plotting the estimate of sensitivity and specificity of above-mentioned biomarkers for diagnosis of NAFLD. (4) There is no restriction on study design. Prospective studies, retrospective studies, and comparative studies were all considered for inclusion. (5) Only full-text articles were included for the meta-analysis.

2.3. Exclusion Criteria. The following studies were excluded: (1) those that were not research articles, including reviews, case reports, and letters to the editors; (2) duplication of records; (3) different articles from a primary study that contained overlapping data cohorts; and (4) the studies associated between biomarkers and NAFLD in vitro or in vivo animal experiments.

2.4. Quality Assessment. The methodology of selected studies was evaluated using Cochrane's Quality Assessment of Diagnostic Accuracy Studies (QUADAS) [36, 37], and the results were demonstrated by using Revman 5.3 (The Nordic Cochrane Centre, Copenhagen, Denmark) with each item scored as "yes," "no," or "unclear." Two authors (LH and LFD) independently reviewed each included study. The disagreement was resolved after discussion.

2.5. Data Extraction. Two independent investigators ( $\mathrm{LH}$ and LFD) carried out the data extraction by a predefined database. The disagreements of these two databases were resolved by joint reevaluation. The following key study characteristics were abstracted from each included study: the first author's name, publication year, study region, study design, numbers of cases and controls, assay method, and accompanying diagnostic thresholds (cut-offs). The number of the true positive, false-positive, false negative, and true negative results was calculated according to liver biopsy and reported cut-off for each diagnosing biomarker. A 0.5 value was automatically added to cells with 0 for adjustment [38].

2.6. Statistical Methods. We used Stata software, version 12.0 (Stata Corp LP, College Station, TX), and Review Manager 5.3 for the meta-analysis; data synthesis was done by using a bivariate approach. The overall diagnostic performance of serum biomarkers was evaluated by using area under the curve on summary receiver operating characteristic curves (AUROC); the combined diagnostic score and diagnostic odds ratio were also used to describe the overall diagnostic ability of the serum biomarkers. Based on $2 \times 2$ diagnostic tables extracted from the included studies, the diagnostic accuracy of the biomarkers was measured by combined sensitivity, specificity, positive likelihood ratio (PLR), and negative likelihood ratio (NLR). Data were presented with 95\% confidence intervals (CIs) and were visualized on forest plots. For heterogeneity assessments, the Cochran $Q$ method was applied and quantified with the $I^{2}$ value. The definitions of heterogeneity were as follows: An $I^{2}$ value less than 50\% was deemed low heterogeneity, and when the $I^{2}$ value was greater than $50 \%$, substantial heterogeneity was considered [36].

\section{Results}

3.1. Literature Search. The detailed manuscript screening processes were shown in Figure 1. Briefly, 1181 articles were 


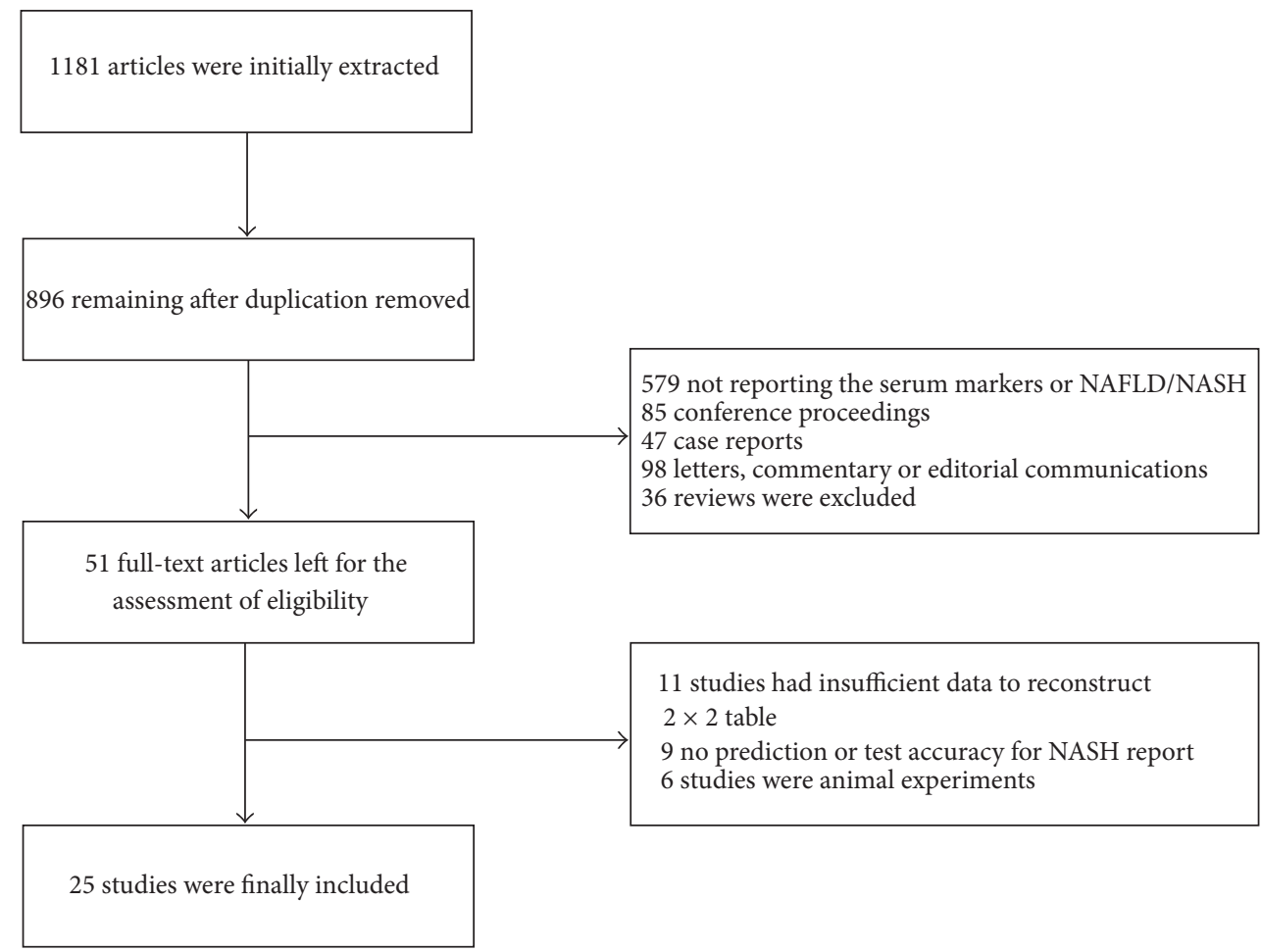

FIGURE 1: Flow diagram demonstrating literature search and selection of studies of CK-18, FGF-21, and combined biomarker panel for diagnosing NASH.

initially identified from above-mentioned four electronic databases. A total of 896 manuscripts that remained after duplicates were removed, among which 845 manuscripts were excluded by reviewing title and abstract for meeting the prespecified exclusion criteria. Of these manuscripts, 579 studies did not report markers or NAFLD/NASH, 85 were conference proceedings, 47 were case reports, 98 were letters, commentary or editorial communications, and 36 were reviews. After full-text assessment of eligible 11 studies contained inadequate data with which to construct a $2 \times$ 2 diagnostic table, nine manuscripts were excluded for not reporting the prediction or accuracy of diagnosis, and six animal experiments were removed. Therefore, a total of 25 manuscripts which presented data on the NAFLD/NASH diagnostic efficiency with CK-18, FGF-21, and combined biomarker panel (CBP) finally met the inclusion criteria and were included in this meta-analysis [11-35].

3.2. Characteristics of Included Studies. Of the 25 included manuscripts, with the publication years spanning 2006 to 2015, 20 were published after 2010, and all 25 articles were published in English. 15 studies involved 1406 patients with data on CK-18, 12 studies involved 1943 patients with data on FGF-21, and seven studies involved 748 patients with data on CBP based on CK-18 and/or FGF-21. All of them are crosssectional studies. Of these 25 studies, six were conducted in China, nine were conducted in United States, and 10 were from Europe. For measurement of serum biomarkers concentrations, all of included studies used blood samples that were tested by ELISA. We provide the characteristics of the 25 manuscripts in Table 1.

The overall methodology quality of the included studies was evaluated by 2 of the authors (LH and LFD) according to QUADAS [37]. All studies met the following applicability concern criteria: the included patients, setting, and reference standard match the quality assessment items; meanwhile, the index test or interpretation was brought into correspondence with the review questions mentioned in QUADAS. In addition, More than $70 \%$ of studies also met items for risk of bias criteria. In this regard, the patient selection was unclear in Musso et al. [16], and three studies [17, 24, 31] also were described as unclear in index test domain. Furthermore, the risk of bias in the reference standard domain was evaluated as high in four studies [16, 22, 27, 31], but it was unclear in Giannini et al. [34] and Yilmaz and Eren [25]. There was a high risk of bias in time and flowing domain in Feldstein et al. [14], Malik et al. [17], and Yilmaz et al. [27], but it was unclear in four studies [11, 12, 20, 22], the details of which were all summarized in Supplementary Figure S1 in Supplementary Material available online at https://doi.org/10.1155/2017/9729107.

3.3. Noninvasive Diagnostic Performance of Circulating Biomarkers. NASH is a potentially serious condition associated with a significant increase in overall and liverrelated morbidity and mortality, because a proportion of patients with NASH develop cirrhosis and HCC $[4,5,39]$. Until now, liver biopsy remains the gold standard for 
TABLE 1: Major characteristics of the studies included in the meta-analysis.

\begin{tabular}{|c|c|c|c|c|c|c|c|c|}
\hline Number & Study (year) & Country & Study design & $\begin{array}{c}\text { Number of } \\
\text { cases }\end{array}$ & $\begin{array}{l}\text { Age, y } \\
\text { (SD) }\end{array}$ & $\begin{array}{c}\text { Gender } \\
(\mathrm{M} / \mathrm{F})\end{array}$ & Methods & Biomarkers \\
\hline 1 & $\begin{array}{l}\text { Wieckowska et al. [11] } \\
\text { (2006) }\end{array}$ & USA & Cross-sectional & 39 & $50.8(11.1)$ & $18 / 21$ & ELISA & CK-18 (M30) \\
\hline 2 & $\begin{array}{l}\text { Yilmaz et al. [12] } \\
\text { (2007) }\end{array}$ & Turkey & Cross-sectional & 83 & $48.9(9.1)$ & $45 / 38$ & ELISA & $\begin{array}{l}\text { CK-18 (M30 \& } \\
\text { M65) }\end{array}$ \\
\hline 3 & Diab et al. [13] (2008) & USA & Cross-sectional & 86 & $48.0(11.1)$ & $38 / 48$ & ELISA & CK-18 (M30) \\
\hline 4 & $\begin{array}{l}\text { Feldstein et al. [14] } \\
\qquad(2009)\end{array}$ & USA & Cross-sectional & 139 & $48.0(1.9)$ & $51 / 88$ & ELISA & CK-18 (M30) \\
\hline 5 & $\begin{array}{l}\text { Papatheodridis et al. } \\
{[15](2010)}\end{array}$ & Greece & Cross-sectional & 58 & $48.0(13.0)$ & $32 / 17$ & ELISA & CK-18 (M30) \\
\hline 6 & $\begin{array}{l}\text { Musso et al. [16] } \\
\text { (2010) }\end{array}$ & Italy & Cross-sectional & 125 & $46.0(4.0)$ & $89 / 36$ & ELISA & CK-18 (M30) \\
\hline 7 & $\begin{array}{l}\text { Malik et al. [17] } \\
\text { (2009) }\end{array}$ & USA & Cross-sectional & 95 & $48.0(5.3)$ & $58 / 37$ & ELISA & CK-18-M30 \\
\hline 8 & Shen et al. [18] (2012) & China & Cross-sectional & 147 & $47.7(9.7)$ & $121 / 99$ & ELISA & $\begin{array}{c}\text { CK-18 (M30 \& } \\
\text { M65) }\end{array}$ \\
\hline 9 & Joka et al. [19] (2012) & Germany & Cross-sectional & 22 & $45.6(3.3)$ & $15 / 7$ & ELISA & $\begin{array}{c}\text { CK-18 (M30 \& } \\
\text { M65) }\end{array}$ \\
\hline 10 & $\begin{array}{l}\text { Younossi et al. [20] } \\
\qquad(2008)\end{array}$ & USA & Cross-sectional & 69 & $41.6(10.6)$ & $23 / 46$ & ELISA & $\begin{array}{c}\text { Combined } \\
\text { biomarker panel }\end{array}$ \\
\hline 11 & $\begin{array}{l}\text { Younossi et al. [21] } \\
\qquad(2011)\end{array}$ & USA & Cross-sectional & 79 & $42.3(10.3)$ & $18 / 61$ & ELISA & $\begin{array}{c}\text { Combined } \\
\text { biomarker panel }\end{array}$ \\
\hline 12 & $\begin{array}{l}\text { Pirvulescu et al. [22] } \\
\qquad(2012)\end{array}$ & Romania & Cross-sectional & 60 & $44.9(9.4)$ & $18 / 42$ & ELISA & $\begin{array}{c}\text { Combined } \\
\text { biomarker panel }\end{array}$ \\
\hline 13 & $\begin{array}{l}\text { Grigorescu et al. [23] } \\
\text { (2012) }\end{array}$ & Romania & Cross-sectional & 79 & $43.7(11.1)$ & $56 / 23$ & ELISA & $\begin{array}{c}\text { Combined } \\
\text { biomarker panel }\end{array}$ \\
\hline 14 & Shen et al. [24] (2012) & China & Cross-sectional & 220 & $48.1(9.7)$ & $121 / 99$ & ELISA & $\begin{array}{c}\text { Combined } \\
\text { biomarker panel }\end{array}$ \\
\hline 15 & $\begin{array}{l}\text { Yilmaz and Eren [25] } \\
\text { (2012) }\end{array}$ & Turkey & Cross-sectional & 136 & $48.8(7.5)$ & $70 / 66$ & ELISA & $\begin{array}{c}\text { Combined } \\
\text { biomarker panel }\end{array}$ \\
\hline 16 & Yang et al. [26] (2015) & China & Cross-sectional & 270 & $30.3(12.7)$ & $128 / 142$ & ELISA & $\begin{array}{c}\text { Combined } \\
\text { biomarker panel }\end{array}$ \\
\hline 17 & $\begin{array}{l}\text { Yilmaz et al. [27] } \\
\qquad(2010)\end{array}$ & Turkey & Cross-sectional & 159 & $47.0(8.0)$ & $71 / 88$ & ELISA & FGF-21 \\
\hline 18 & Li et al. [28] (2010) & China & Cross-sectional & 348 & $43.5(11.1)$ & $212 / 136$ & ELISA & FGF-21 \\
\hline 19 & $\begin{array}{l}\text { Dushay et al. [29] } \\
\text { (2010) }\end{array}$ & Spain & Cross-sectional & 21 & $31(10.0)$ & $7 / 14$ & ELISA & FGF-21 \\
\hline 20 & $\begin{array}{l}\text { Dasarathy et al. [30] } \\
\text { (2011) }\end{array}$ & USA & Cross-sectional & 26 & $43.3(7.0)$ & $13 / 13$ & ELISA & FGF-21 \\
\hline 21 & $\begin{array}{l}\text { Reinehr et al. [31] } \\
\qquad(2012)\end{array}$ & USA & Cross-sectional & 60 & $12.0(1.4)$ & $30 / 30$ & ELISA & FGF-21 \\
\hline 22 & Li et al. [32] (2013) & China & Cross-sectional & 712 & $51.4(12.9)$ & $278 / 434$ & ELISA & FGF-21 \\
\hline 23 & $\begin{array}{l}\text { Shen Y et al. [33] } \\
\text { (2013) }\end{array}$ & China & Cross-sectional & 74 & $63.9(8.6)$ & $39 / 35$ & ELISA & FGF-21 \\
\hline 24 & $\begin{array}{l}\text { Giannini et al. [34] } \\
\text { (2013) }\end{array}$ & USA & Cross-sectional & 217 & $15.0(0.4)$ & $91 / 126$ & ELISA & FGF-21 \\
\hline 25 & Alisi et al. [35] (2013) & Italy & Cross-sectional & 107 & $10.5(4.8)$ & $41 / 66$ & ELISA & FGF-21 \\
\hline
\end{tabular}

$\mathrm{SD}$, standard deviation. M, male. F, female.

diagnosing and grading NASH. However, it is obvious that this invasive procedure is not suitable as a screening test for such a prevalent condition, and this in turn restricts new promising therapies currently being tested in large clinical trials [40]. For all of these reasons, there is an urgent need to develop and validate simple, reproducible, noninvasive tests that accurately distinguish NASH from NAFLD and determine the stage and grade of the disease. 
3.4. CK-18. CK-18 is a major intermediate filament protein in hepatocytes. Apoptosis of hepatocytes is further associated with the release of caspase-cleaved and uncleaved CK-18 fragments, namely, M30 and M65 [41]. In this study, a total of 15 studies reported concentrations of CK-18 fragments including M30 and M65 in patients who developed NASH (Table 1).

3.5. Meta-Analysis on Group M30. For the including studies, the cut-offs chosen ranged 121.6-380.2 U/L (0.60-0.95 sensitivity and $0.60-0.97$ specificity). The AUROC for these M30 testing studies ranged 0.66-0.93 (Supplementary Table $\mathrm{S} 1)$. The combined diagnostic score and diagnostic odds ratio were 2.33 (95\% CI, 1.80-2.86) and 10.30 (95\% CI, 6.07-17.49), respectively (Figure 2 ). The combined sensitivity, specificity, PLR, and NLR of M30 were 0.75 (95\% CI, 0.69-0.81), 0.77 (95\% CI, 0.68-0.84), 3.28 (95\% CI, 2.32-4.65), and 0.32 (95\% CI, 0.25-0.41), respectively (Supplementary Figure S2). In addition, the AUROC value of the M30 testing in predicting NASH was 0.82 (95\% CI, 0.79-0.85), showing ability to distinguish NASH from NAFLD (Figure 3).

3.6. Meta-Analysis on Group M65. In the including 6 studies, the cut-offs ranged $243.8-790 \mathrm{U} / \mathrm{L}(0.62-1$ sensitivity and 0.65-0.89 specificity) and the AUROC for these studies ranged 0.71-0.93 (Supplementary Table S2). According to the reported concentrations of M65, the summary diagnostic score and diagnostic odds ratio were 2.12 (95\% CI, 1.51-2.72) and 8.31 (95\% CI, 4.55-15.19), respectively (Supplementary Figure S3). This corresponds to a combined sensitivity, specificity, PLR, and NLR of 0.71 (95\% CI, 63\%-78\%), 0.77 (95\% CI, 0.67-0.84), 3.09 (95\% CI, 2.11-4.54), and 0.37 (95\% CI, 0.28-0.49), respectively (Supplementary Figure S4). As the AUROC value of M65 was 0.80 (95\% CI, 0.76-0.83) (Supplementary Figure S5), this indicated that M65 and M30 testing had similar performance to distinguish NASH from simple steatosis. However, its overall diagnostic accuracy and stability need to be verified by more research data.

3.7. FGF-21. FGF-21, a liver-secret hormone, has recently been shown to possess beneficial effects on lipid metabolism and hepatic steatosis [42]. Several studies demonstrated that serum FGF-21 concentrations were associated with hepatic fat content especially in subjects with moderate hepatic steatosis $[28,43,44]$. In this meta-analysis, there were 12 studies reporting on the testing of FGF-21 concentrations in NAFLD patients (Table 1 and Supplementary Table S3).

3.8. Meta-Analysis on FGF-21. The concentrations of FGF21 of the patient who developed NAFLD were significantly higher than control group; the summary standardized mean difference (SMD) was 1.37 (95\% CI, 0.54-2.21) (Supplementary Figure S6). Particularly, in the subgroup analysis performed between simple steatosis group and NASH group, the SMD was more remarkable (SMD 1.47, 95\% CI, 0.13-3.07) in NASH than the studies that tested from simple steatosis (SMD 1.12, 95\% CI 0.27-1.97) (Supplementary Figure S7). These results suggest that the serum FGF-21 can be potentially used as a biomarker for NAFLD/NASH.
Then, we further identified four studies that pooled the sensitivity and specificity of FGF-21 testing on NASH. The diagnostic score, diagnostic odds ratio, sensitivity, and specificity were 1.74 (95\% CI, 1.22-2.26), 5.70 (95\% CI, 3.389.62), 0.62 (95\% CI, 0.50-0.73), and 0.78 (95\% CI, 0.70-0.84), respectively (Figure 4 ). However, we did not extract enough data from included studies to estimate the AUROC value of FGF-21.

3.9. CBP. The development of NAFLD is related to inflammation, hepatocyte apoptosis, and fibrosis during disease progression [45]. Meanwhile, different noninvasion biomarkers, such as CK-18, FGF-21, and IL-1Ra, involved in NAFLD progression were significantly correlated with NAS score and the pathological characteristics of NAFLD [26]. Importantly, some reviews recently indicated that combining two or more individual biomarkers as a panel could obtain a better predictive value for NASH $[7,8,46]$.

3.10. Meta-Analysis on CBP. The diagnostic accuracy of the biomarker panel for NASH was reported in seven studies. In these studies, as shown in Supplementary Table S4, the CBP was designed including CK-18, FGF-21, and other different markers.

The AUROC value of the CBP was 0.94 (95\% CI, 0.920.96), compared to CK-18 or FGF-21 assay, which showed the most significant ability to distinguish NASH from simple steatosis (Figure 5). The combined diagnostic score and diagnostic odds ratio of the CBP were 4.17 (95\% CI, 3.31-5.02) and 64.48 (95\% CI, 27.39-151.78), respectively (Figure 6(a)). The combined sensitivity and specificity were 0.92 (95\% CI, 0.880.95 ) and 0.85 (95\% CI, 0.72-0.92), respectively (Figure 6(b)).

3.11. Test of Cross-Study Heterogeneity. There was marked cross-study heterogeneity in the CK-18 fragment M30, FGF21, and the CBP in pooled diagnostic score, sensitivity, specificity, PLR, and NLR; the $I^{2}$ values were shown in Supplementary Table S5, whereas moderate heterogeneity was observed in the CK-18 fragment M65 combined diagnostic score, specificity, PLR, and NLR; the $I^{2}$ values were $42.89 \%$, $43.52 \%, 0.00 \%$, and $38.13 \%$, respectively.

\section{Discussion}

In view of the remarkable increase in prevalence of NAFLD in conjunction with the significant research effort in developing novel therapies for patients with $\mathrm{NASH}$, noninvasion, convenient, reproducible, and reliable serum biomarkers are greatly needed. However, due to technical and accuracy issues, the noninvasion biomarkers for diagnosis of NAFLD, especially distinguishing NASH from NAFLD, have not been widely used all over the world.

In this systematic review, we investigated evidence for diagnostic capability of serum noninvasion biomarkers in NAFLD. Over all, the findings from the meta-analysis indicate that upregulated levels of CK-18 and FGF-21 in serum are associated with increased risk for NASH. Particularly, the diagnostic panels which combined with several biomarkers including CK-18 and/or FGF-21 showed excellent 

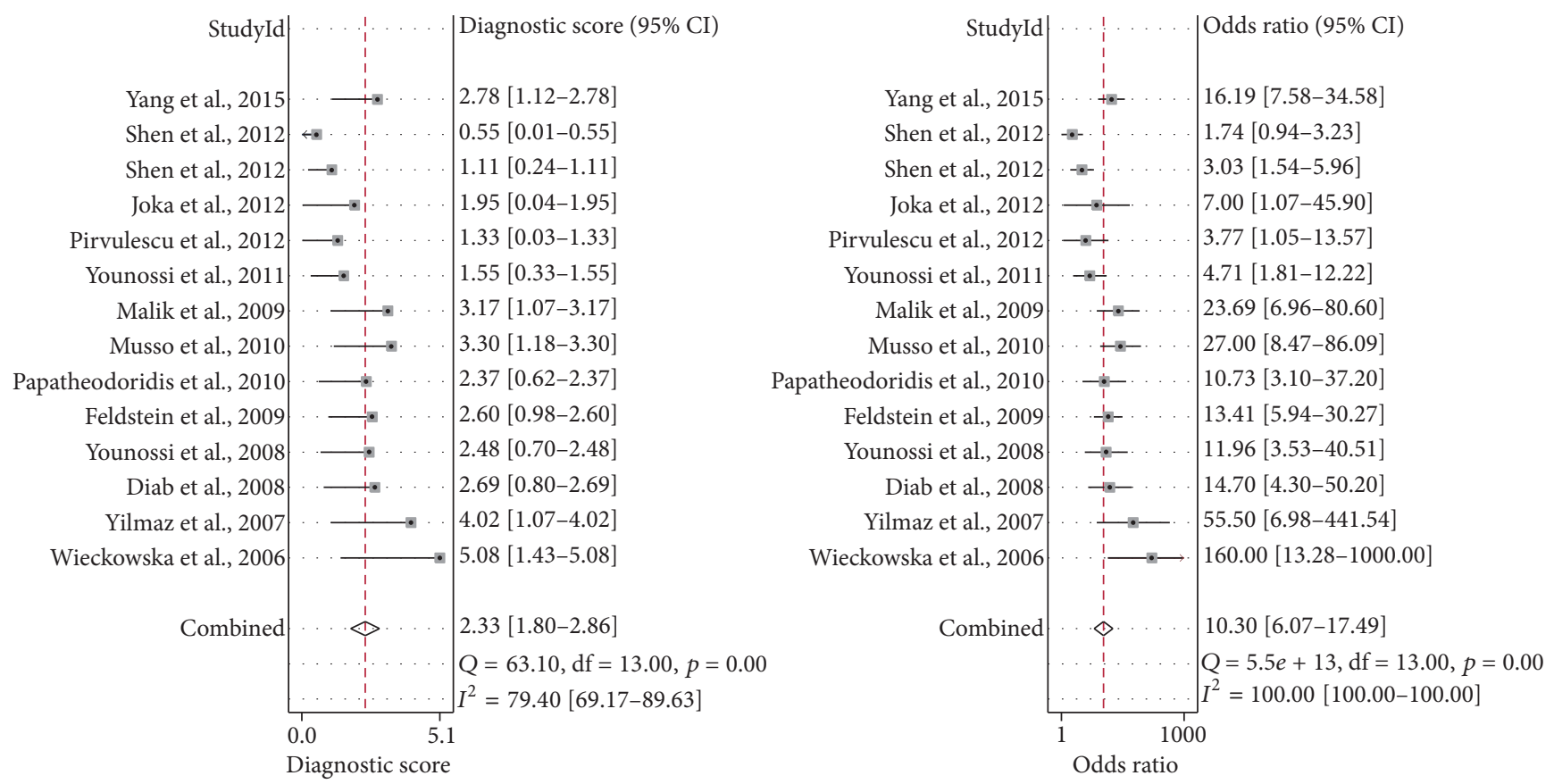

FIgURE 2: Combined DS and DOR of CK-18, M30 fragment. DS, diagnostic score. DOR, diagnostic odds ratio. CI, confidence interval.



FIGURE 3: Summary receiver's operative characteristics of CK-18, M30 fragment. AUC, area under the curve. SENS, sensitivity. SPEC, specificity. 

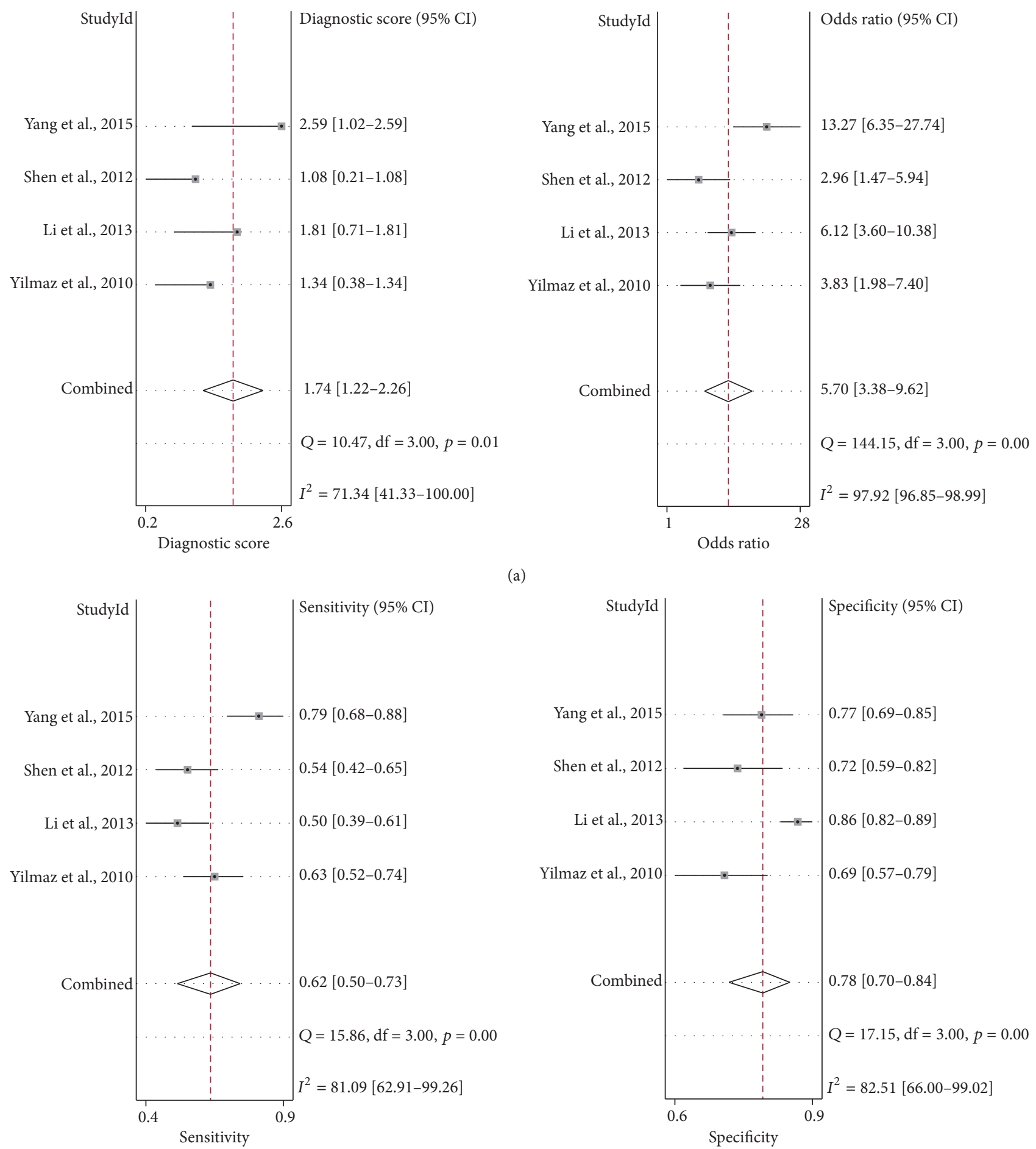

(a)

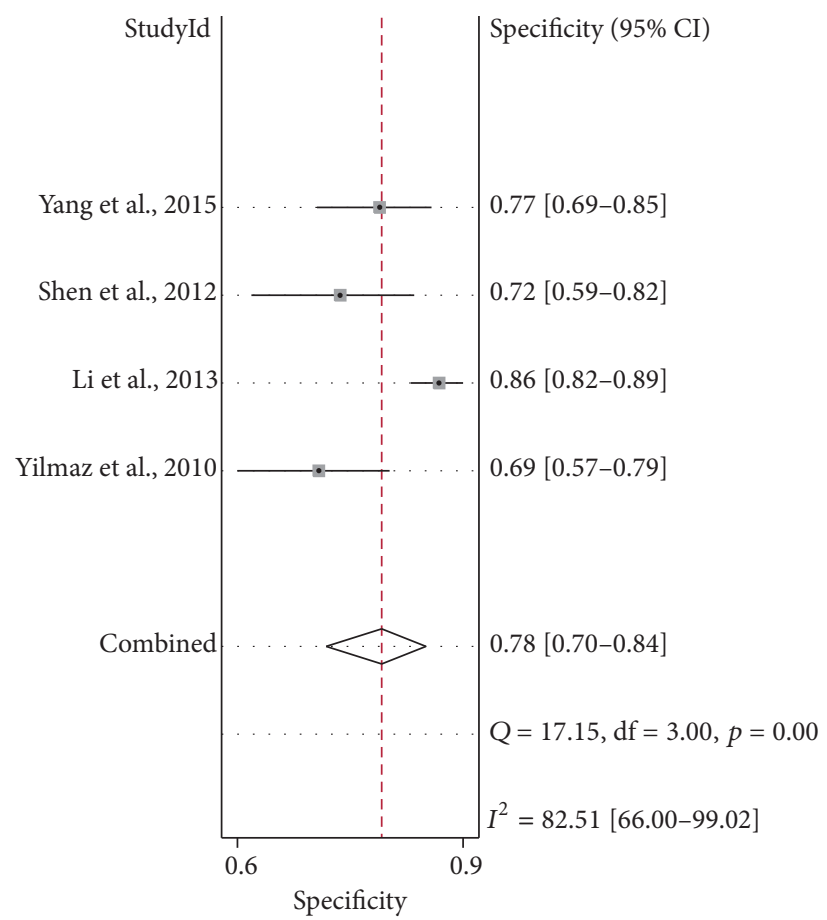

(b)

FIGURE 4: Combined DS, DOR (a), and sensitivity and specificity (b) of FGF-21. DS, diagnostic score. DOR, diagnostic odds ratio. CI, confidence interval.

performance for distinguishing NASH from NAFLD. To our knowledge, this is the first ever meta-analysis in which diagnostic value of the most studied principal biomarkers is compared simultaneously in NAFLD patients. Despite the increasing number of studies and reviews concerning serum noninvasion biomarkers for NAFLD, there is no consensus regarding which biomarkers have best diagnostic value. Only a few systematic reviews evaluated the diagnostic capability of CK-18 based on its sensitivity and specificity $[9,10]$ but did not summarize and compare with other similar biomarkers. 


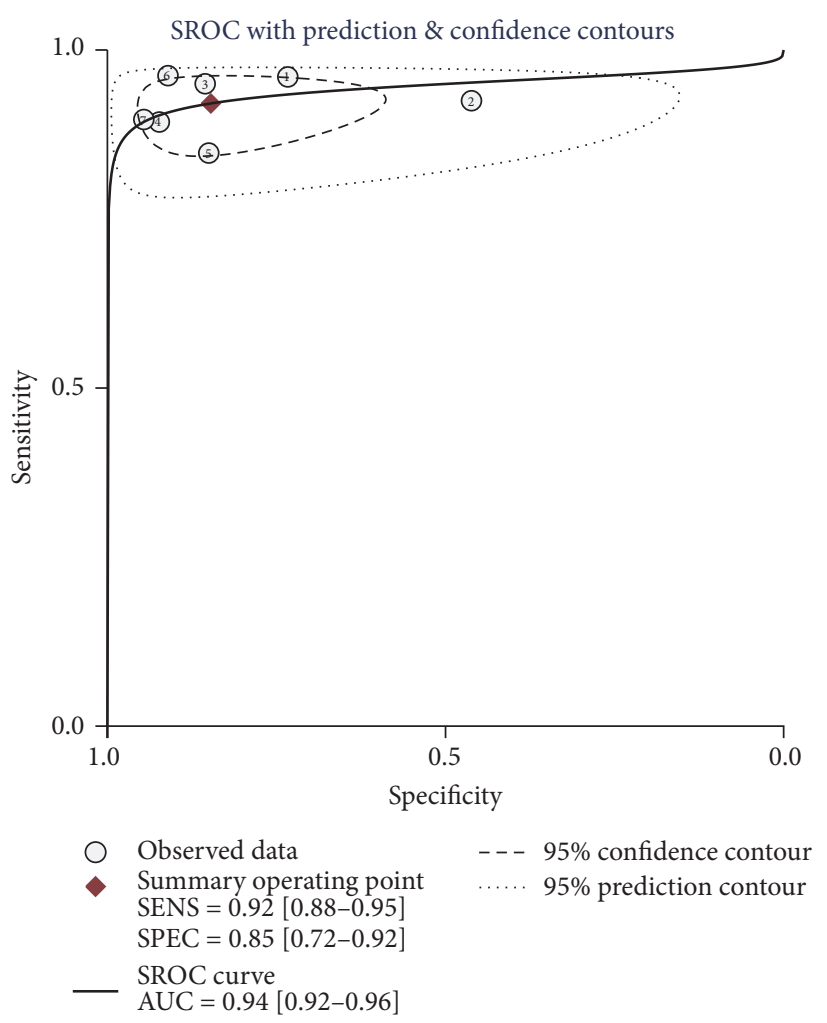

FIGURE 5: Summary receiver's operative characteristics of CBP. AUC, area under the curve. SENS, sensitivity. SPEC, specificity.

As is well known, laboratory tests that are routinely included in the evaluation of patients with suspected NAFLD include a serum panel of liver tests (alanine aminotransferase (ALT), aspartate aminotransferase, alkaline phosphatase, and gamma-glutamyl-transpeptidase). However, Mofrad et al. [47] demonstrated that the entire histological spectrum of NAFLD can be seen in patients with normal ALT values. Moreover, Kunde et al. [48] also evaluated the diagnostic accuracy of serum ALT for NASH diagnosis, which was found to be quite poor, at merely $40 \%$. Given the limitations of serum transaminases as noninvasive selective indicators of NAFLD, overwhelming evidence showed that the certain cytokines derived from several biochemical events including insulin resistance, oxygen stress, apoptosis, or inflammation may play more important roles in the progression of NAFLD [49]. For instance, during hepatocyte apoptosis, the fragments of CK-18 can be detectable in serum of chronic liver diseases patients by ELISA [50], and this method was tested as a promising noninvasive tool in NASH diagnosis. Serum FGF-21 was also associated with liver fat content and damage, which could be the useful circulating biomarker for predicting progression in NAFLD patients [34]. Furthermore, adipose tissue contributes to NAFLD, being a source of fatty acids and cytokines such as adiponectin, imbalance of which seems to be associated with severe NAFLD $[51,52]$. The data from Wree et al. further indicated that reduced adiponectin levels may establish a proinflammatory milieu, thus increasing vulnerability to lipotoxicity, exacerbating hepatocytes injury, which promotes progression from simple steatosis to NASH and even advanced hepatic fibrosis [53].
Importantly, there is general consensus that a PLR of greater than 10 and a NLR of less than 0.05 provide reliable evidence of satisfactory diagnostic performance [54]. Based on our meta-analysis, none of the above-mentioned biomarkers fulfilled the criteria to be able to satisfactorily discriminate between patients with NASH versus NAFLD. However, the overall results suggest that CK-18 (M30) has moderate accuracy for diagnosing NASH (0.75 sensitivity, 0.77 specificity). This means $75 \%$ suspected NASH patients will be identified by CK-18 and avoid a liver biopsy, while $23 \%$ of patients who were initially diagnosed as non-NASH according to CK-18 still require a liver biopsy for further identification. Also, CK-18 (M30) with NLR of 0.32 indicates a higher risk of missing NASH. Meanwhile, CK-18 (M65) is more likely a useful biomarker for identifying NASH rather than screening, due to significant homogeneity observed in pooled specificity and PLR among the studies. Furthermore, CK-18 has some additional advantage over other biomarkers. One of the reasons is the fact that CK-18 is a major component of intermediate filaments of hepatocytes, and the circulating fragment of CK-18 can specifically reflect the degree of hepatocellular apoptosis, which is a characteristic of NASH [55].

As compared to CK-18, FGF-21 improves insulin sensitivity and insulin resistance in obesity animal models [56]. Several studies also suggest that elevated serum FGF-21 is likely due to time-dependent expression of Fgf21 mRNA in human hepatocytes, which is more related to unsaturated fatty acids but is opposite to the patterns of insulin and glucose [43, 57, 58]. Our findings suggest that FGF-21 showed excellent 



(a)

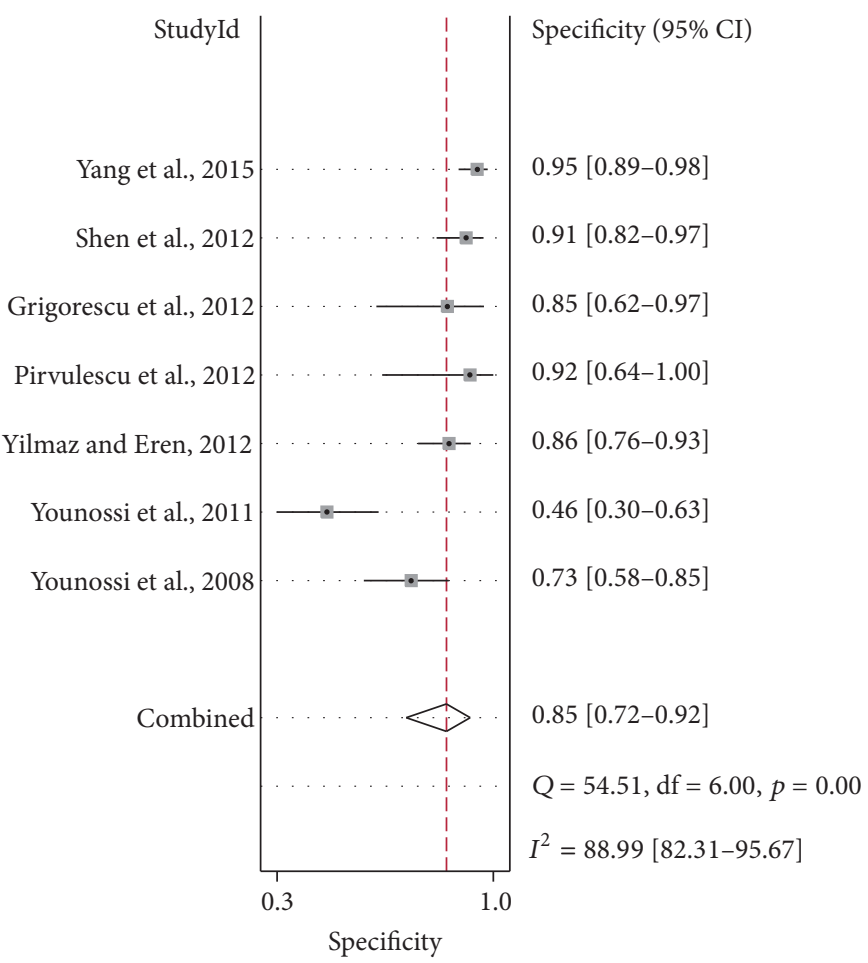

(b)

Figure 6: Pooled DS, DOR (a), and sensitivity and specificity (b) of CBP. DS, diagnostic score. DOR, diagnostic odds ratio. CI, confidence interval.

performance to distinguish NASH from hepatic steatosis. With a combined specificity of 0.78 , FGF-21 was good at identifying NASH. Nevertheless, its ability to confirm the diagnosis was inadequate due to the fact that the number of studies included is very few. Only four studies met the inclusive criteria, which contained adequate data to construct the diagnostic table. Also, there is some heterogeneity among the studies. These two factors weaken the conclusion. However, 
these results provide clues about the role of FGF-21 as a key regulator of hepatic lipid metabolism in humans and suggest that serum FGF-21 can be used as a biomarker for NASH.

The most promising application of some of these novel biomarkers for the detection of simple steatosis and NASH may be in the combination of several into diagnostic panels. In this study, we evaluated the diagnosis effect with the CBP. With the pooled AUROC of 0.94, the biomarker panel showed the most excellent diagnostic performance for diagnosis of NASH. Its pooled sensitivity and specificity were 0.92 and 0.85 in contrast to those of CK-18 or FGF-21, which had the highest sensitivity and specificity. Therefore, during noninvasive diagnosis and monitoring NASH procedure, when CK-18 or FGF-21 initially diagnoses NASH, additional steps with other biomarkers should be performed in case of false-positive results.

The remarkable cross-study heterogeneity was found in this meta-analysis, which may have been due to factors like methodology quality, country, study design, and sample size. In general, the heterogeneity was more evident in the results with the circulating level of noninvasive biomarker measured by the $I^{2}$ value. In the absence of a standardized operation introduction, features like the storage conditions of blood sample, antibody titer for different biomarkers, and instrument accuracy are likely to be the source of heterogeneity in included studies.

There were several limitations in this study, the most important of which was derived from the different methodologies. First, few biomarkers met the rigorous criteria for diagnostic test accuracy, suggesting that if such diagnostic test accuracy assessments were available, the present conclusions could be markedly affected. Furthermore, although the results of this meta-analysis showed that combined biomarker panel performed well for NASH diagnostic, the high $I^{2}$ value indicated marked heterogeneity in included studies; a subgroup analysis according to the degree of NASH could not be conducted owing to inadequate data. With the emergence of numerous new studies about CBP, we will update the meta-analysis in further study.

\section{Conclusion}

As the first meta-analysis to comprehensively and quantitatively evaluate the relationship between noninvasive biomarkers and NAFLD, this study demonstrates that CK18, FGF-21, and related biomarker panel can be used to diagnose NAFLD, especially NASH. Importantly, use of CBP resulted in improved accuracy with highest sensitivity and specificity, when compared to use of single biomarker including CK-18 and FGF-21. Further research is required to validate the most optimized pooled biomarker panel in a well-structured, population-based cohort study with blinded evaluation. Additionally, understanding how abovementioned biomarkers influence NASH progression may help to elucidate potentially biological mechanisms for determining treatment strategies and prognosis.

\section{Competing Interests}

The authors declare no competing financial interests.

\section{Authors' Contributions}

Lei He, Linfeng Deng, and Wenjian Song conceived the study design; Lei He and Linfeng Deng collected and analyzed the data; Jianli Guo served as a consultant in statistical analysis of data; Quan Zhang and Jinan Zhou rechecked the results; Lei He and Fahu Yuan wrote the first draft of the manuscript and all the authors contributed in the analysis of data and preparation of the final version of the manuscript. All the authors approved the final version of the manuscript. Lei He and Linfeng Deng contributed equally to this work.

\section{Acknowledgments}

This work was supported by the grant from the National Natural Science Foundation of China (no. 81300304).

\section{References}

[1] G. Marchesini, E. Bugianesi, G. Forlani et al., "Nonalcoholic fatty liver, steatohepatitis, and the metabolic syndrome," Hepatology, vol. 37, no. 4, pp. 917-923, 2003.

[2] E. Fabbrini, S. Sullivan, and S. Klein, "Obesity and nonalcoholic fatty liver disease: biochemical, metabolic, and clinical implications," Hepatology, vol. 51, no. 2, pp. 679-689, 2010.

[3] S. Chitturi, G. C. Farrell, and J. George, "Non-alcoholic steatohepatitis in the Asia-Pacific region: future shock?" Journal of Gastroenterology and Hepatology (Australia), vol. 19, no. 4, pp. 368-374, 2004.

[4] G. A. Michelotti, M. V. Machado, and A. M. Diehl, "NAFLD, NASH and liver cancer," Nature Reviews Gastroenterology \& Hepatology, vol. 10, no. 11, pp. 656-665, 2013.

[5] M. S. Ascha, I. A. Hanouneh, R. Lopez, T. A.-R. Tamimi, A. F. Feldstein, and N. N. Zein, "The incidence and risk factors of hepatocellular carcinoma in patients with nonalcoholic steatohepatitis," Hepatology, vol. 51, no. 6, pp. 1972-1978, 2010.

[6] J. Ertle, A. Dechêne, J.-P. Sowa et al., "Non-alcoholic fatty liver disease progresses to hepatocellular carcinoma in the absence of apparent cirrhosis," International Journal of Cancer, vol. 128, no. 10, pp. 2436-2443, 2011.

[7] A. Wieckowska, A. J. McCullough, and A. E. Feldstein, "Noninvasive diagnosis and monitoring of nonalcoholic steatohepatitis: present and future," Hepatology, vol. 46, no. 2, pp. 582-589, 2007.

[8] D. E. Amacher, "Progress in the search for circulating biomarkers of nonalcoholic fatty liver disease," Biomarkers, vol. 19, no. 7, pp. 541-552, 2014.

[9] R. Kwok, Y.-K. Tse, G. L.-H. Wong et al., "Systematic review with meta-analysis: non-invasive assessment of non-alcoholic fatty liver disease-the role of transient elastography and plasma cytokeratin-18 fragments," Alimentary Pharmacology and Therapeutics, vol. 39, no. 3, pp. 254-269, 2014.

[10] J. Chen, Y. Y. Zhu, Q. Zheng, and J. J. Jiang, "Serum cytokeratin18 in the diagnosis of non-alcoholic steatohepatitis: a metaanalysis," Hepatology Research, vol. 44, no. 8, pp. 854-862, 2014.

[11] A. Wieckowska, N. N. Zein, L. M. Yerian, A. R. Lopez, A. J. McCullough, and A. E. Feldstein, "In vivo assessment of 
liver cell apoptosis as a novel biomarker of disease severity in nonalcoholic fatty liver disease," Hepatology, vol. 44, no. 1, pp. 27-33, 2006.

[12] Y. Yilmaz, E. Dolar, E. Ulukaya et al., "Soluble forms of extracellular cytokeratin 18 may differentiate simple steatosis from nonalcoholic steatohepatitis," World Journal of Gastroenterology, vol. 13, no. 6, pp. 837-844, 2007.

[13] D. L. Diab, L. Yerian, P. Schauer et al., "Cytokeratin 18 fragment levels as a noninvasive biomarker for nonalcoholic steatohepatitis in bariatric surgery patients," Clinical Gastroenterology and Hepatology, vol. 6, no. 11, pp. 1249-1254, 2008.

[14] A. E. Feldstein, A. Wieckowska, A. R. Lopez, Y.-C. Liu, N. N. Zein, and A. J. McCullough, "Cytokeratin-18 fragment levels as noninvasive biomarkers for nonalcoholic steatohepatitis: a multicenter validation study," Hepatology, vol. 50, no. 4, pp. 1072-1078, 2009.

[15] G. V. Papatheodoridis, E. Hadziyannis, E. Tsochatzis et al., "Serum apoptotic caspase activity in chronic hepatitis C and nonalcoholic fatty liver disease," Journal of Clinical Gastroenterology, vol. 44, no. 4, pp. E87-E95, 2010.

[16] G. Musso, R. Gambino, M. Durazzo, and M. Cassader, "Noninvasive assessment of liver disease severity with liver fat score and CK-18 in NAFLD: prognostic value of liver fat equation goes beyond hepatic fat estimation," Hepatology, vol. 51, no. 2, pp. 715-717, 2010.

[17] R. Malik, M. Chang, K. Bhaskar et al., "The clinical utility of biomarkers and the nonalcoholic steatohepatitis CRN liver biopsy scoring system in patients with nonalcoholic fatty liver disease," Journal of Gastroenterology and Hepatology, vol. 24, no. 4, pp. 564-568, 2009.

[18] J. Shen, H. L.-Y. Chan, G. L.-H. Wong et al., "Assessment of non-alcoholic fatty liver disease using serum total cell death and apoptosis markers," Alimentary Pharmacology \& Therapeutics, vol. 36, no. 11-12, pp. 1057-1066, 2012.

[19] D. Joka, K. Wahl, S. Moeller et al., "Prospective biopsycontrolled evaluation of cell death biomarkers for prediction of liver fibrosis and nonalcoholic steatohepatitis," Hepatology, vol. 55, no. 2, pp. 455-464, 2012.

[20] Z. M. Younossi, M. Jarrar, C. Nugent et al., "A novel diagnostic biomarker panel for obesity-related nonalcoholic steatohepatitis (NASH)," Obesity Surgery, vol. 18, no. 11, pp. 1430-1437, 2008.

[21] Z. M. Younossi, S. Page, N. Rafiq et al., "A biomarker panel for non-alcoholic steatohepatitis (NASH) and NASH-related fibrosis," Obesity Surgery, vol. 21, no. 4, pp. 431-439, 2011.

[22] I. Pirvulescu, L. Gheorghe, I. Csiki et al., "Noninvasive clinical model for the diagnosis of nonalcoholic steatohepatitis in overweight and morbidly obese patients undergoing bariatric surgery," Chirurgia (Bucharest, Romania : 1990), vol. 107, no. 6, pp. 772-779, 2012.

[23] M. Grigorescu, D. Crisan, C. Radu, M. D. Grigorescu, Z. Sparchez, and A. Serban, "A novel pathophysiologicalbased panel of biomarkers for the diagnosis of nonalcoholic steatohepatitis," Journal of Physiology and Pharmacology, vol. 63, no. 4, pp. 347-353, 2012.

[24] J. Y. Shen, H. L.-Y. Chan, G. L.-H. Wong et al., "Non-invasive diagnosis of non-alcoholic steatohepatitis by combined serum biomarkers," Journal of Hepatology, vol. 56, no. 6, pp. 1363-1370, 2012.

[25] Y. Yilmaz and F. Eren, "Identification of a support vector machine-based biomarker panel with high sensitivity and specificity for nonalcoholic steatohepatitis," Clinica Chimica Acta, vol. 414, pp. 154-157, 2012.
[26] M. Yang, D. Xu, Y. Liu et al., "Combined serum biomarkers in non-invasive diagnosis of non-alcoholic steatohepatitis," PLOS ONE, vol. 10, no. 6, Article ID e0131664, 2015.

[27] Y. Yilmaz, F. Eren, O. Yonal et al., "Increased serum FGF21 levels in patients with nonalcoholic fatty liver disease," European Journal of Clinical Investigation, vol. 40, no. 10, pp. 887-892, 2010.

[28] H. T. Li, Q. C. Fang, F. Gao et al., "Fibroblast growth factor 21 levels are increased in nonalcoholic fatty liver disease patients and are correlated with hepatic triglyceride," Journal of Hepatology, vol. 53, no. 5, pp. 934-940, 2010.

[29] J. Dushay, P. C. Chui, G. S. Gopalakrishnan et al., "Increased fibroblast growth factor 21 in obesity and nonalcoholic fatty liver disease," Gastroenterology, vol. 139, no. 2, pp. 456-463, 2010.

[30] S. Dasarathy, Y. Yang, A. J. McCullough, S. Marczewski, C. Bennett, and S. C. Kalhan, "Elevated hepatic fatty acid oxidation, high plasma fibroblast growth factor 21, and fasting bile acids in nonalcoholic steatohepatitis," European Journal of Gastroenterology \& Hepatology, vol. 23, no. 5, pp. 382-388, 2011.

[31] T. Reinehr, J. Woelfle, R. Wunsch, and C. L. Roth, "Fibroblast Growth Factor 21 (FGF-21) and its relation to obesity, metabolic syndrome, and nonalcoholic fatty liver in children: a longitudinal analysis," Journal of Clinical Endocrinology \& Metabolism, vol. 97, no. 6, pp. 2143-2150, 2012.

[32] H. Li, K. Dong, Q. Fang et al., "High serum level of fibroblast growth factor 21 is an independent predictor of non-alcoholic fatty liver disease: a 3-year prospective study in China," Journal of Hepatology, vol. 58, no. 3, pp. 557-563, 2013.

[33] Y. Shen, X. Ma, J. Zhou et al., "Additive relationship between serum fibroblast growth factor 21 level and coronary artery disease," Cardiovascular Diabetology, vol. 12, no. 1, article 124, 2013.

[34] C. Giannini, A. E. Feldstein, N. Santoro et al., "Circulating levels of FGF-21 in obese youth: associations with liver fat content and markers of liver damage," Journal of Clinical Endocrinology \& Metabolism, vol. 98, no. 7, pp. 2993-3000, 2013.

[35] A. Alisi, S. Ceccarelli, N. Panera et al., "Association between serum atypical fibroblast growth factors 21 and 19 and pediatric nonalcoholic fatty liver disease," PLoS ONE, vol. 8, no. 6, Article ID e67160, 2013.

[36] N. Panic, E. Leoncini, G. De Belvis, W. Ricciardi, and S. Boccia, "Evaluation of the endorsement of the preferred reporting items for systematic reviews and meta-analysis (PRISMA) statement on the quality of published systematic review and meta-analyses," PLOS ONE, vol. 8, no. 12, 2013.

[37] P. F. Whiting, A. W. S. Rutjes, M. E. Westwood et al., "Quadas-2: a revised tool for the quality assessment of diagnostic accuracy studies," Annals of Internal Medicine, vol. 155, no. 8, pp. 529-536, 2011.

[38] H. Sahai and A. Khurshid, "On analysis of epidemiological data involving a $2 \times 2$ contingency table: an overview of fisher's exact test and yates' correction for continuity," Journal of Biopharmaceutical Statistics, vol. 5, no. 1, pp. 43-70, 1995.

[39] A. Wree, L. Broderick, A. Canbay, H. M. Hoffman, and A. E. Feldstein, "From NAFLD to NASH to cirrhosis-new insights into disease mechanisms," Nature Reviews Gastroenterology \& Hepatology, vol. 10, no. 11, pp. 627-636, 2013.

[40] I. Nalbantoglu and E. M. Brunt, "Role of liver biopsy in nonalcoholic fatty liver disease," World Journal of Gastroenterology, vol. 20, no. 27, pp. 9026-9037, 2014.

[41] H. Bantel, P. Ruck, M. Gregor, and K. Schulze-Osthoff, "Detection of elevated caspase activation and early apoptosis in liver 
diseases," European Journal of Cell Biology, vol. 80, no. 3, pp. 230-239, 2001.

[42] Y. C. Woo, A. M. Xu, Y. Wang, and K. S. L. Lam, "Fibroblast Growth Factor 21 as an emerging metabolic regulator: clinical perspectives," Clinical Endocrinology, vol. 78, no. 4, pp. 489-496, 2013.

[43] F. M. Fisher, P. C. Chui, P. J. Antonellis et al., "Obesity is a fibroblast growth factor 21 (FGF21)-resistant state," Diabetes, vol. 59, no. 11, pp. 2781-2789, 2010.

[44] H. Yan, M. Xia, X. Chang et al., "Circulating fibroblast growth factor 21 levels are closely associated with hepatic fat content: a cross-sectional study," PLoS ONE, vol. 6, no. 9, 2011.

[45] G. C. Farrell and C. Z. Larter, "Nonalcoholic fatty liver disease: from steatosis to cirrhosis," Hepatology, vol. 43, no. 2, pp. S99S112, 2006.

[46] Y. Yilmaz, E. Ulukaya, and E. Dolar, "A 'biomarker biopsy' for the diagnosis of NASH: promises from CK-18 fragments," Obesity Surgery, vol. 18, no. 11, pp. 1507-1508, 2008.

[47] P. Mofrad, M. J. Contos, M. Haque et al., "Clinical and histologic spectrum of nonalcoholic fatty liver disease associated with normal ALT values," Hepatology, vol. 37, no. 6, pp. 1286-1292, 2003.

[48] S. S. Kunde, A. J. Lazenby, R. H. Clements, and G. A. Abrams, "Spectrum of NAFLD and diagnostic implications of the proposed new normal range for serum ALT in obese women," Hepatology, vol. 42, no. 3, pp. 650-656, 2005.

[49] A. J. Sanyal, "Mechanisms of disease: pathogenesis of nonalcoholic fatty liver disease," Nature Clinical Practice Gastroenterology and Hepatology, vol. 2, no. 1, pp. 46-53, 2005.

[50] H. Hetz, K. Hoetzenecker, S. Hacker et al., "Caspase-cleaved cytokeratin 18 and $20 \mathrm{~S}$ proteasome in liver degeneration," Journal of Clinical Laboratory Analysis, vol. 21, no. 5, pp. 277281, 2007.

[51] A. Wree, A. Kahraman, G. Gerken, and A. Canbay, "Obesity affects the liver-the link between adipocytes and hepatocytes," Digestion, vol. 83, no. 1-2, pp. 124-133, 2011.

[52] A. Wree, L. Broderick, A. Canbay, H. M. Hoffman, and A. E. Feldstein, "From NAFLD to NASH to cirrhosis-new insights into disease mechanisms," Nature Reviews Gastroenterology and Hepatology, vol. 10, no. 11, pp. 627-636, 2013.

[53] A. Wree, M. Schlattjan, L. P. Bechmann et al., "Adipocyte cell size, free fatty acids and apolipoproteins are associated with non-alcoholic liver injury progression in severely obese patients," Metabolism: Clinical and Experimental, vol. 63, no. 12, pp. 1542-1552, 2014.

[54] P. Eusebi, "Diagnostic accuracy measures," Cerebrovascular Diseases, vol. 36, no. 4, pp. 267-272, 2013.

[55] P. Valva, E. De Matteo, M. C. Galoppo, M. I. Gismondi, and M. V. Preciado, "Apoptosis markers related to pathogenesis of pediatric chronic hepatitis $\mathrm{C}$ virus infection: M30 mirrors the severity of steatosis," Journal of Medical Virology, vol. 82, no. 6, pp. 949-957, 2010.

[56] J. Xu, D. J. Lloyd, C. Hale et al., "Fibroblast growth factor 21 reverses hepatic steatosis, increases energy expenditure, and improves insulin sensitivity in diet-induced obese mice," Diabetes, vol. 58, no. 1, pp. 250-259, 2009.

[57] T. Inagaki, P. Dutchak, G. Zhao et al., "Endocrine regulation of the fasting response by ppar $\alpha$-mediated induction of fibroblast growth factor 21," Cell Metabolism, vol. 5, no. 6, pp. 415-425, 2007.
[58] H. Y. Yu, F. Z. Xia, K. S. L. Lam et al., "Circadian rhythm of circulating fibroblast growth factor 21 is related to diurnal changes in fatty acids in humans," Clinical Chemistry, vol. 57, no. 5, pp. 691-700, 2011. 


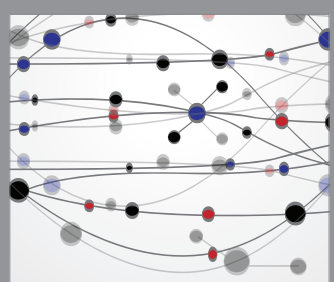

The Scientific World Journal
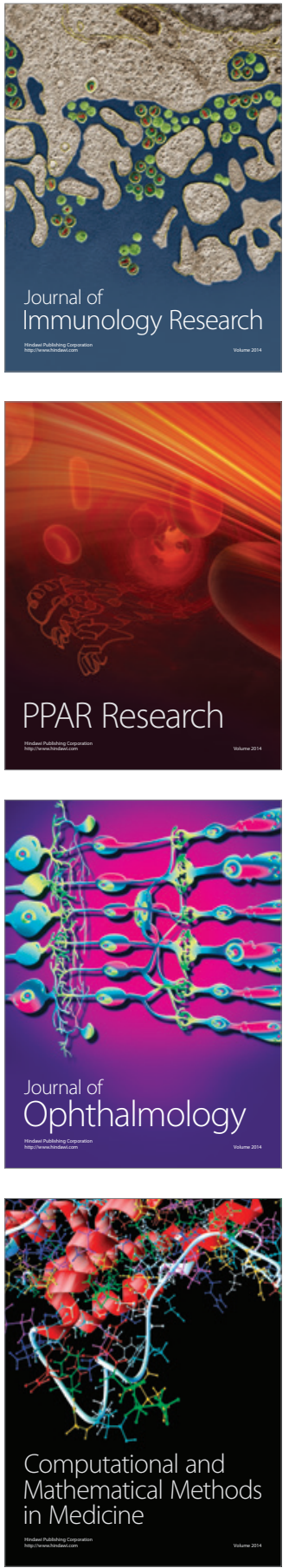

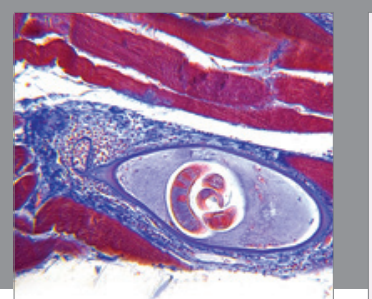

Gastroenterology Research and Practice
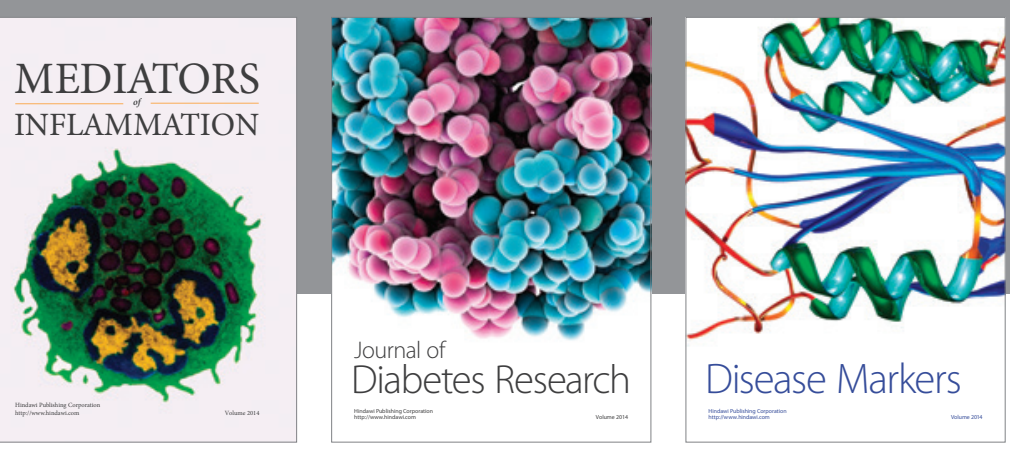

Disease Markers



\section{Hindawi}

Submit your manuscripts at

https://www.hindawi.com
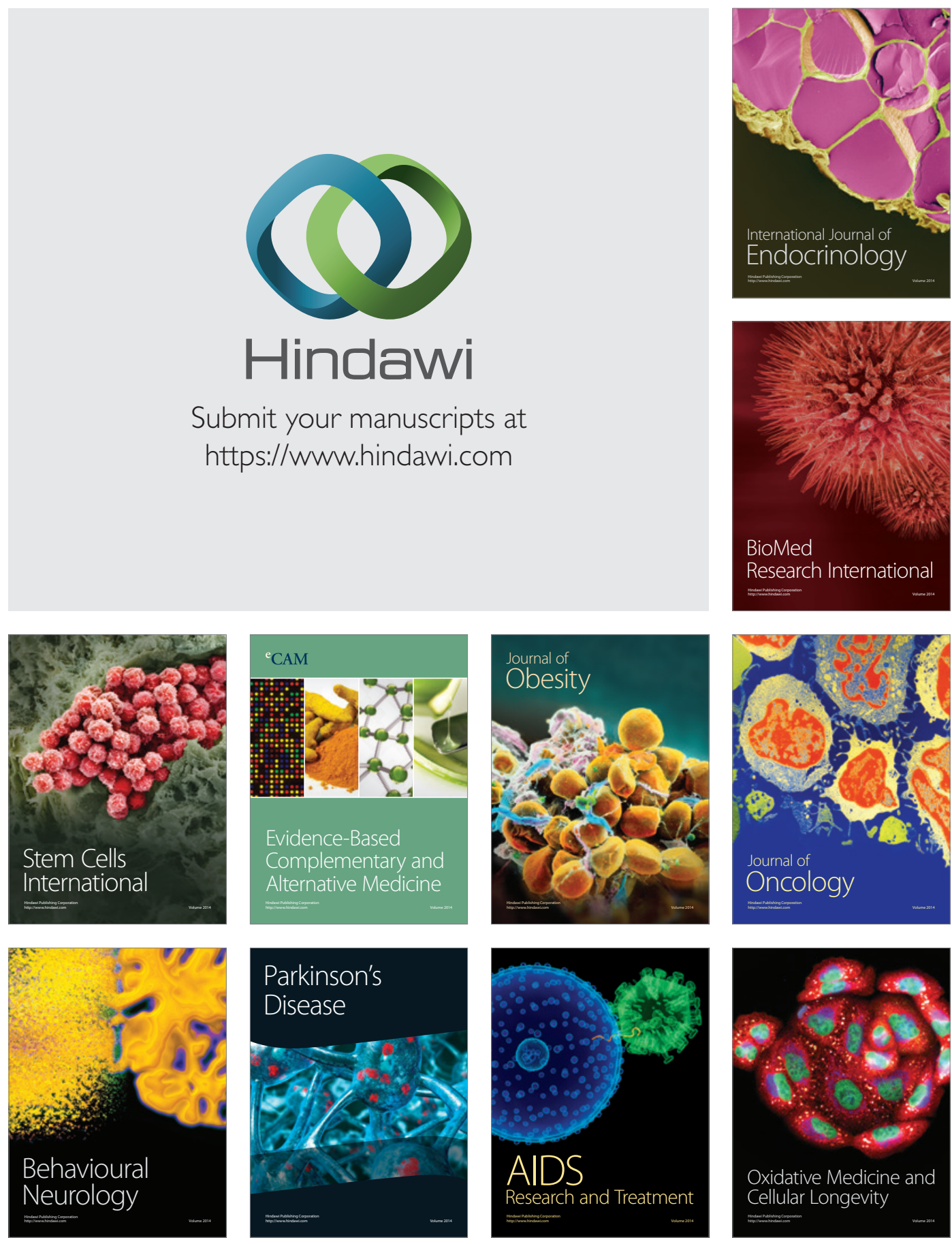